\title{
FUSIÓN, COEDICION O REESTRUCTURACIÓN DE REVISTAS CIENTÍFICAS EN HUMANIDADES Y CIENCIAS SOCIALES
}

Luis Rodríguez-Yunta y Elea Giménez-Toledo

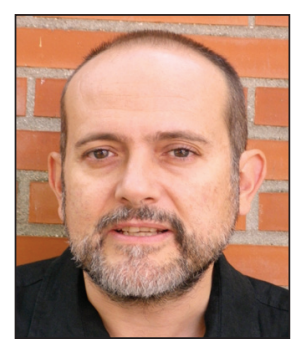

Luis Rodríguez-Yunta es documentalista en el CSIC desde 1986 y forma parte del equipo de trabajo de la base de datos ISOC en el Centro de Ciencias Humanas y Sociales (CCHS). Tiene titulación de doctor en documentación (2011) por la Universidad Complutense de Madrid, en cuya Facultad de Documentación ha sido profesor asociado entre 2007 y 2010. Es colaborador del Grupo EPUC y secretario ejecutivo de la Red Europea de Documentación e Información sobre América Latina (Redial).

http://orcid.org/0000-0002-8424-6205

luis.ryunta@cchs.csic.es

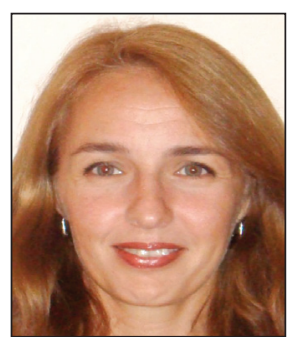

Elea Giménez-Toledo es científica titular del CSIC. Licenciada y doctora en documentación, es responsable del G.I. de Evaluación de Publicaciones Científicas (EPUC) del Centro de Ciencias Humanas y Sociales. Su actividad investigadora se centra en los procesos de evaluación de la actividad científica en ciencias humanas y sociales. Además, diseña y aplica indicadores de calidad para revistas científicas y editoriales de libros. Es coautora de las plataformas de evaluación de revistas $D I C E, R E S H$ y CIRC y es responsable de la parte española de Latindex. También es promotora del sistema de indicadores para editoriales SPI (Scholarly Publisher's Indicators).

http://orcid.org/0000-0001-5425-0003

elea.gimenez@cchs.csic.es

Centro de Ciencias Humanas y Sociales (CCHS) Consejo Superior de Investigaciones Científicas (CSIC) Albasanz, 26-28. 28037 Madrid, España

\section{Resumen}

Se analizan las debilidades del sector editorial de revistas de Humanidades y Ciencias sociales en España. Predominan las publicaciones de carácter institucional o generadas por un pequeño colectivo (asociación, departamento, grupo de investigación) con una baja producción anual, escaso impacto, gestión poco eficaz y que encuentra dificultades para superar cierta imagen de endogamia. Frente a este modelo dominante, se plantea la fusión de publicaciones, la coedición o la restructuración de las revistas actuales para mejorar en su gestión y encontrar un mayor reconocimiento nacional e internacional.

\section{Palabras clave}

Revistas científicas, Gestión editorial, Evaluación de revistas, Ciencias sociales, Ciencias humanas.

\section{Title: Fusion, copublication or restructuring research journals in the humanities and social sciences}

\begin{abstract}
This paper analyzes the weaknesses of the Humanities and Social Sciences journal publishing sector in Spain. A high percentage of these journals are published by universities and often as a result of the effort of a small team (e.g., professional association, department, research group). They often have very low numbers of papers published per year, limited impact, and inefficient management, and may find it difficult to overcome a certain image of inbreeding. Against this dominant model, the authors propose the merger, co-publishing or restructuring of current journals to improve their management and achieve greater national and international recognition.
\end{abstract}

\section{Keywords}

Scholarly journals, Editorial management, Journal evaluation, Social sciences, Humanities.

Rodríguez-Yunta, Luis; Giménez-Toledo, Elea (2013). “Fusión, coedición o reestructuración de revistas científicas en humanidades y ciencias sociales". El profesional de la información, enero-febrero, v. 22, n. 1, pp. 36-45. 


\section{Introducción}

La producción editorial de revistas de Humanidades y Ciencias sociales (en adelante HHyCS) en España se encuentra dispersa en un número excesivo de títulos que en muchas ocasiones no alcanzan un suficiente eco en su propia comunidad científica. Los datos de edición de revistas no se corresponden con el peso real de las disciplinas humanísticas y sociales en los sistemas de docencia e investigación. A modo de ejemplo, la estructura del CSIC otorga a estas disciplinas sólo 1 de sus 8 áreas científicas (13\%), mientras que en su plataforma web Revistas CSIC le corresponden 26 de un total de 37 (70\%). Igualmente la Universidad Complutense de Madrid dedica a las HHyCS 68 de las 76 revistas presentes en su plataforma editorial Revistas científicas complutenses (89\%) frente a 11 de sus 21 facultades $(52 \%)^{1}$. http://revistas.csic.es

En España se publican muchas más revistas de HHyCS que de Ciencia, Tecnología y Medicina. El sector editorial predominante es el de la Universidad. Ésta acumula el $42 \%$ de las publicaciones periódicas en estas disciplinas, mientras que sólo suponen el $17 \%$ de las de Ciencia y Tecnología según los directorios de las bases de datos del CSIC. En segundo lugar destacan las revistas editadas por asociaciones y fundaciones que en el caso de las HHyCS representan un $23 \%$ y en el caso de la Ciencia y la Tecnología es bastante mayor: $44 \%$ (tabla 1).

De acuerdo con estos datos, un 79\% de las publicaciones españolas de HHyCS estarían editadas por organizaciones sin ánimo de lucro, un porcentaje muy superior al que señala Morris (2007) con respecto al mismo concepto en la edición científica mundial: $55 \%$.

La dispersión de publicaciones en estas áreas no afecta exclusivamente a España. Se ha señalado que la cobertura de revistas del Ulrich's periodical directory en los índices de Web of science es mucho menos representativa en estas disciplinas que en ciencias naturales y tecnológicas (Archambault et al., 2006). Entre otras muchas razones, esto puede ser un reflejo de las dificultades encontradas para delimitar las publicaciones de la corriente principal en estas disciplinas. Los datos de Latindex ${ }^{2}$ indican que en España la situación es especialmente exacerbada: un $71 \%$ de los registros de publicaciones españolas en este directorio son de HHyCS, mientras en Brasil, por ej., suponen el $58 \%$.

Entre las debilidades del sector destaca la gran cantidad de publicaciones con sólo uno o dos números al año. En mu- chos casos la preparación de cada número es un ejercicio voluntarista realizado por un pequeño equipo o, incluso, por una sola persona. La herencia del pasado ha fomentado la revista "institucional" gestionada por departamentos universitarios que, a menudo, no puede asumir un mayor esfuerzo en su realización. O bien se trata de iniciativas ligadas a la imagen exterior de instituciones culturales, organismos o asociaciones, que difícilmente pueden considerarse representativas de una disciplina en su globalidad.

Si bien en los últimos años se ha extendido la concienciación sobre la necesidad de mejorar la calidad, aún ha de producirse una transformación radical en el sector (OliveraBetrán, 2011). Ante las tendencias que se vislumbran: edición digital, acceso abierto, mayor especialización, creciente internacionalización tanto en la estructura de su comité científico como en la autoría y apertura a la interactividad de las redes sociales, cabe preguntarse si la fusión de títulos y la coedición contribuirían a un importante cambio para superar la imagen predominante, personalista y amateur.

Muchas revistas son iniciativas individuales, de grupos de investigación, departamentos, museos, pequeñas asociaciones... con un escaso número de investigadores

\section{Objetivos}

En el presente trabajo se analizan las debilidades actuales de la edición de revistas de HHyCS en España y se plantean como posibles alternativas la restructuración o la concentración de algunas de las publicaciones. En segundo lugar, se presentan las ventajas e inconvenientes de esta posible fusión, y se revisan algunas experiencias de éxito en este sentido.

\section{Metodología}

Se hace una revisión crítica a partir del análisis de las revistas científicas españolas en HHyCS y la participación en seminarios, congresos y conferencias especializadas sobre su calidad y evaluación. Los datos se han extraído fundamentalmente de tres fuentes:

- Directorio de revistas de Ciencias sociales y Humanidades, ligado a la base de datos ISOC dentro de la plataforma de bases de datos bibliográficas del $\mathrm{CSIC}^{3}$, producida por el Centro de Ciencias Humanas y Sociales. En esta fuente se recogen 2.712 registros de publicaciones españolas, de los cuales 1.880 son títulos vivos.

- La base de datos interna de revistas del Grupo de Investigación de Evaluación de Publicaciones Científicas (EPUC) del Centro de Ciencias Humanas y Sociales del CSIC, que reúne 
2.147 revistas, de las cuales 1.778 son títulos vivos.

- El sistema de evaluación de revistas científicas Resh, que toma como fuente la base de datos mencionada anteriormente y ofrece indicadores de distinta naturaleza para todas sus publicaciones.

http://epuc.cchs.csic.es/resh

\section{Análisis de debilidades del sector editorial de las revistas españolas de HHyCS}

\subsection{Predominio de un modelo no profesional en la gestión editorial}

La gestión de un gran número de publicaciones está marcada por un fuerte carácter personalista desde su propio nacimiento. Muchas revistas son iniciativas individuales, de grupos de investigación, departamentos, museos, pequeñas asociaciones, centros culturales o institutos con un escaso número de investigadores. La toma real de decisiones se concentra en muy pocas manos. En este modelo de gestión, la dirección de la publicación es un cargo prácticamente vitalicio ejercido por su "fundador", hasta el punto de que la revista desaparece cuando su "alma mater" se retira, o cambia de sede institucional cuando el director se traslada.

Gran parte de las iniciativas de edición generalmente seleccionan sólo artículos sobre temas de investigación que coincidan con las líneas de trabajo del colectivo fundador y tienen una marcada orientación interna, esto es, se han creado como canal de difusión de la actividad del colectivo, incluyen noticias de sus actividades o incluso su memoria anual. Estos contenidos podrían ubicarse mejor en otro tipo de publicaciones pero no en revistas científicas en las que la publicación de artículos de investigación original debe ser lo predominante. En el contexto de la evaluación de la actividad científica, una revista de estas características difícilmente puede aspirar a un reconocimiento de primer nivel.

Otra característica ligada a la no profesionalización de la gestión es una producción anual muy limitada: a menudo se publica un único número al año, y son frecuentes retrasos en la publicación. En la base de datos ISOC, se recogen 20.890 artículos publicados en $2010^{4}$, que proceden de 1.013 títulos de revistas. De estas publicaciones 502 (49,6\%) no superan los 15 registros en dicho año, y 685 (67,6\%) no sobrepasan los 20 documentos. Este handicap se produce igualmente en las publicaciones de mayor prestigio. De las 25 revistas de $\mathrm{HHyCS}$ presentes en la plataforma editorial del CSIC, 15 no alcanzaron la media de 20 artículos anuales y solamente 2 superaron los 30 para el período 2010-2011. http://revistas.csic.es

Se percibe una cierta confusión sobre qué tipo de contenidos tienen cabida en una revista como vehículo de comunicación científica de calidad. Aunque formalmente se afirma aplicar un sistema de libre presentación de trabajos y revisión anónima de los mismos, en la práctica se aplican con frecuencia modos de trabajo idénticos a los utilizados para elaborar obras colectivas: selección previa de autores a los que se propone una colaboración, personas que asumen el rol de coordinadores de un monográfico y que también son autores de alguno de los artículos, o incluso estructura en forma de capítulos resultado de un reparto

\begin{tabular}{|l|c|c|}
\hline \multicolumn{1}{|c|}{ Autores externos } & Revistas vivas & $\%$ \\
\hline Más del $80 \%$ & 706 & 40,3 \\
\hline Entre 60 y $79 \%$ & 346 & 19,7 \\
\hline Entre 40 y 59\% & 126 & 7,2 \\
\hline Entre 20 y 39\% & 48 & 2,7 \\
\hline Menos del 20\% & 25 & 1,4 \\
\hline Sin valor calculado ${ }^{5}$ & 503 & 28,7 \\
\hline Total & 1.754 & 100,0 \\
\hline
\end{tabular}

Tabla 2: Distribución de las revistas de HHyCS en función del porcentaje de autores externos. Fuente: base de datos interna del grupo EPUC, abril 2012

previo de aspectos de un tema monográfico. Así por ejemplo, de las 25 revistas de HHyCS presentes en la plataforma editorial del CSIC, 13 incluyeron algún dossier o monográfico en 2010-2011. Sea por falta de financiación para producir monografías o por confusión del rol de la revista, lo cierto es que a menudo se utilizan éstas para suplir otro tipo de publicación: actas de congresos, seminarios y jornadas de conferencias, compilaciones en homenaje a una figura señera, informes de excavaciones o proyectos, etc.

Una de las críticas a las revistas que más preocupa a los editores es la de su carácter endogámico (Cantó-Alcaraz, 2008; Casals-Carro, 2011; Editor de Formación Universitaria, 2012), aunque se procura evitar esta imagen promoviendo la publicación de trabajos de diferentes procedencias, a fin de superar el indicador tenido en cuenta por los sistemas de evaluación de revistas y por las agencias, por ejemplo Cneai (Ministerio de Educación, 2011) o Anep (Anep/Fecyt, 2007). Sin embargo, siguen presentándose algunas características que apuntan a cierta endogamia en los procedimientos editoriales:

a) Publicación frecuente de los autores del centro editor. Aunque no llegan al 50\% de los artículos de un número, sí es habitual la presencia de artículos elaborados por alguno de los miembros de los comités editoriales o por personas adscritas al mismo departamento o grupo de investigación que controla la edición. Como puede observarse en la tabla 2, aún son muchas las revistas cuyo grado de apertura al exterior es insuficiente. Claro está que estos porcentajes dependen del tamaño de la comunidad científica a la que va dirigida pero también es verdad que, precisamente en las disciplinas o especialidades más pequeñas, proliferan los títulos de revistas que luego no logran captar originales de autores externos a la institución editora.

b) Números monográficos coordinados por un investigador en el que escriben sólo personas que colaboran en el mismo proyecto de investigación además del propio coordinador. Por ejemplo, de los 39 dosieres o monográficos incluidos en 2010-2011 en revistas de HHyCS de la plataforma editorial del CSIC, en 25 casos el compilador del mismo participaba también con un artículo.

Otro aspecto a considerar es la existencia de publicaciones fundadas por un investigador o un pequeño grupo, que no cuentan con presencia en la plataforma web del servicio de publicaciones de su institución. De ello se infiere que se trata de iniciativas editoriales personales, desarrolladas en el tiempo de trabajo de un miembro de la institución pero 
que no están enmarcadas en su política editorial. En las plataformas creadas por los servicios de publicaciones de las universidades y centros de investigación hay ausencias notables de títulos que, sin embargo, pueden estar alojados en servidores de la propia institución. Pueden considerarse como revistas no respaldadas (y no financiadas, apoyadas en su proceso técnico, etc.) por el servicio de publicaciones de la institución.

\subsection{Consecuencias negativas sobre la difusión y reco- nocimiento de las revistas}

Una de las quejas más frecuentes por parte de los editores en los foros de revistas científicas es la falta de reconocimiento de estos esfuerzos editoriales y su dificultad para entrar en las principales bases de datos internacionales. Como afirma Pérez-Padilla (2006) para referirse a las revistas médicas latinoamericanas: "Tenemos demasiadas revistas, y todas tienen dentro de sus objetivos obtener su ingreso al famoso Index medicus on line (Medline) y al Science citation index del Institute of Scientific Information (hoy Thomson Reuters). No se requiere mucha investigación para tener claro que es un imposible para la gran mayoría de ellas". $Y$ sin embargo las publicaciones nacionales pueden tener más visibilidad que las internacionales para la comunicación científica en aspectos como la práctica y la gestión médica (Bordons, 2004).

En el caso de las HHyCS, la dispersión de iniciativas es evidente como también lo es la escasa solidez de muchas de ellas. Las principales debilidades identificadas del sector editorial son:

- Escasa estabilidad de las listas de publicaciones de una disciplina, con continuas altas de nuevos títulos y bajas de otros que desaparecen o sufren largos períodos de inactividad.

- Limitado número de citas recibidas por cada publicación, más acentuado aún si se eliminan las autocitas ${ }^{6}$. Por poner sólo algunos ejemplos: de las 41 revistas presentes en Resh para la categoría Historia moderna y contemporánea, 13 no han recibido ninguna cita en el período 2004-2008. Lo mismo ocurre con 90 de las 188 revistas de Educación editadas en España.

- Falta de originales, que llevan a admitir textos con escaso rigor o interés, por necesidad de seguir publicando números, aunque de igual forma se incumpla la periodicidad. Un $24 \%$ de las revistas (423 títulos según los datos recogidos en la base interna del grupo EPUC) no publican sus correspondientes números en el plazo indicado por ellas mismas.

- Reticencias para publicar la lista de evaluadores. Si bien es cierto que estas listas pueden permitir identificar al evaluador de cada artículo en comunidades científicas pequeñas, también es cierto que esta práctica es una muestra de transparencia en relación con la selección de manuscritos.

- Como consecuencia de todo esto, y teniendo en cuenta además las limitaciones en los fondos de las bibliotecas para adquirir revistas, estas publicaciones reciben escasas suscripciones y no tienen presencia en los foros académicos y profesionales.
Es necesario resaltar la idea de que las agencias de evaluación de la actividad científica exigen la publicación en revistas extranjeras de impacto, por la necesidad evidente de internacionalizar la investigación realizada en España. La producción científica de cada investigador en un año, por poner un ejemplo, es limitada y si ha de priorizar la publicación en revistas extranjeras, aún pierde más sentido el hecho de que se sigan multiplicando las revistas españolas pues parece obvio que tendrán un problema de recepción de originales.

La fusión de publicaciones es una alternativa para superar el modelo de revista imperante en $\mathrm{HHyCS}$

Las dificultades para la evaluación científica de las HHyCS han sido desencadenantes de trabajos específicos sobre las revistas científicas españolas de estas áreas (Osca-Lluch; Mateo-Marquina, 2003; Delgado-López-Cózar et al., 2005; Esteban-Navarro et al., 2005; Borrego; Urbano, 2006; Alcaín-Partearroyo; Román-Román; Giménez-Toledo, 2008; Giménez-Toledo; Román-Román; Alcaín-Partearroyo, 2007; Mañana-Rodríguez; Giménez-Toledo, 2011; RuizPérez; Delgado-López-Cózar; Jiménez-Contreras, 2006; Torres-Salinas; Delgado-López-Cózar; Jiménez-Contreras, 2009). Al presentar datos para un análisis de la situación y al establecer indicadores de calidad indirectos se corre el riesgo de que algunos editores modifiquen de manera artificial el valor de esos indicadores y, por otra parte, de que se malinterprete el sentido de los sistemas de evaluación que se construyen. Aportar indicadores para la totalidad de revistas de un país o de una región no debe confundirse nunca con un reconocimiento de buena calidad de todas las publicaciones allí incluidas. Herramientas como Latindex, Dice, Resh, In-Recs, In-Recj, Carhus Plus+ o Miar se crean, y así deben utilizarse, para definir las características de todas y cada una de las revistas que se editan, de tal forma que lo importante no es estar en esos sistemas sino cómo se está, qué nivel de calidad alcanzan las revistas según indicadores de calidad indirectos diseñados y aplicados con metodología científica. Para una institución no es relevante aparecer con numerosas publicaciones en estos sistemas sino que las revistas que estén editando -aunque sean pocas- tengan buena calidad, sean competitivas y estén profesionalizadas.

Este hecho queda olvidado a menudo cuando se presentan datos cuantitativos sin análisis, cayendo en el error de presentar una perspectiva "nacionalista" o "regional" de producción editorial en función del lugar de edición, que no aporta verdadero valor a los estudios. En España se han analizado en ocasiones las revistas en función de si son editadas en determinada región (Osca-Lluch; Mateo-Marquina, 2003; Osca-Lluch et al., 2008); sin embargo, aunque estos datos ayudan a conocer la "demografía" del sector editorial, la mayor o menor producción de revistas no es en sí misma un factor relevante. Por otra parte se deslizan errores por simplificación: las publicaciones de organismos internacionales quedan clasificadas por su sede central; y las de redes de colaboración se adscriben a una única sede que puede 


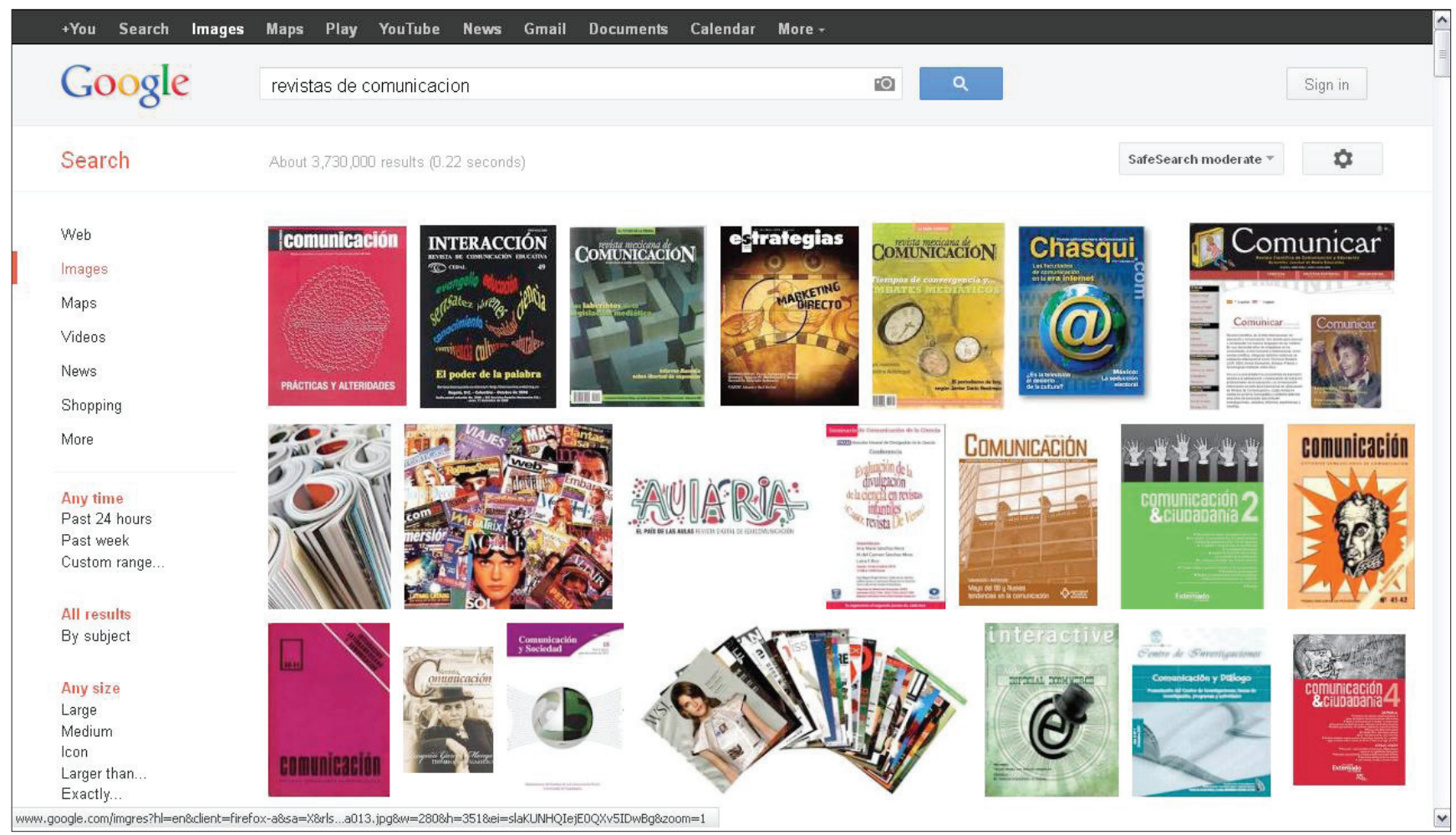

Búsqueda de revistas de comunicación en Google Images

ser meramente coyuntural. El registro numérico de títulos ofrece la falsa impresión de que las cosas van mejor cuantas más revistas haya, como si se tratara de un ranking por presencia en el campo editorial. La proliferación de títulos de revistas no debería ser un dato en sí mismo positivo para la institución o la región que las publica si no va acompañada de la correspondiente calidad.

Las iniciativas para establecer sistemas de categorización de las revistas científicas por parte de las agencias de evaluación (Anep/Fecyt, 2007) y diferentes grupos de investigación (Circ, Carhus, y sistemas de evaluación como Resh, Dice, In-Recs, etc.), también están teniendo cierta influencia (Torres-Salinas et al., 2010; Delgado-López-Cózar et al., 2005; Alcaín-Partearroyo; Román-Román; Giménez-Toledo, 2008). A partir de la toma de conciencia sobre el respeto a las pautas de edición científica consideradas como "buenas prácticas" a nivel internacional, muchas publicaciones han realizado cambios con el objetivo de mejorar su reconocimiento externo, y gracias a ello, algunos títulos han logrado introducirse en los índices internacionales (Mañana-Rodríguez; Giménez-Toledo, 2011). Especialmente se ha realizado un esfuerzo por incluir autores y miembros de los comités asesores procedentes de distintas instituciones y de distintos países, y por incorporar procesos de revisión por expertos para todos los originales. Si bien es cierto que algunos de los cambios producidos son reales, profundos y transformadores de las revistas, también se han detectado casos en los que este proceso de reacción a las crecientes exigencias hacia las revistas ha conllevado meros cambios formales, cuando no prácticas que otros editores y evaluadores denuncian:

- ¿Funcionan los equipos de redacción y comités asesores? ¿Colaboran los miembros externos en las funciones que se presuponen para un comité o se limitan a figurar? ¿Se reúnen los comités, son operativos y tienen incidencia real sobre el contenido? (Giménez-Toledo et al., 2009).

- ¿Se trata de aumentar artificialmente el factor de impacto?

- ¿Funciona siempre el peer review? ¿'es todo lo abierto/ externo que debería ser? ¿afecta igual a todo el contenido de la revista?

Los sistemas de evaluación de revistas intentan a su vez reaccionar y corregir, en la medida de lo posible, las malas prácticas. Por ejemplo, se han establecido ciertas penalizaciones -fundamentalmente, la exclusión temporal de las revistas en los índices de citas- para las publicaciones con malas prácticas de citación, pero aún puede haber cierta incidencia de la autocitación, de modo que es necesario su seguimiento a través del tiempo.

Por otra parte, también se reclama que las publicaciones hagan públicos los porcentajes de rechazo. Pocas revistas ofrecen datos sobre ello y las que lo hacen los dan en bruto sin especificar los motivos de rechazo, por lo que estas informaciones resultan difíciles de relacionar con la calidad de los contenidos publicados.

Estas dificultades para la evaluación no son exclusivas de las HHyCS, pero también contribuyen a frenar los esfuerzos del sector por elevar su prestigio científico.

\section{Fusión y coedición como alternativas para mejorar la imagen de las revistas españolas de HHyCS}

Los sistemas de categorización de revistas están alentando un importante esfuerzo colectivo por la mejora de la calidad de las publicaciones (Cetto et al., en prensa). Los editores deben ser conscientes de que lo importante no es "estar" en estos sistemas, sino "cómo se está". Desde la perspectiva de 
la edición de revistas científicas es necesario distinguir entre calidad y excelencia (Guédon, 2011). Calidad y profesionalidad son imprescindibles para la excelencia, pero no son el único factor. Si se aspira a la calidad basta con cumplir unas pautas consensuadas como buenas prácticas, está al alcance de cualquier editor. Por el contrario, si se aspira a la excelencia, es necesario ejercer una posición central en la comunicación científica dentro de una comunidad, garantizar visibilidad, reconocimiento y credibilidad. La excelencia no está al alcance de todos y es un objetivo al que difícilmente se puede dirigir una publicación que nace centrada en intereses institucionales o con claras debilidades estructurales.

El voluntarismo en la gestión y la dispersión de esfuerzos define un panorama en el que resulta muy difícil mejorar la imagen colectiva de la producción científica en HHyCS y aspirar a la excelencia. Si las revistas se sostienen sólo en base a la entrega no reconocida de pequeños equipos, no es posible garantizar la pervivencia de muchas de las publicaciones actuales, más aún en un escenario de grave crisis económica como el que estamos afrontando. Por ello resulta conveniente que exista un núcleo central de revistas en las diferentes disciplinas que sean consideradas como representativas del área del conocimiento y que faciliten la publicación de los mejores trabajos sin ningún tipo de discriminación en función de su adscripción institucional. La fusión de publicaciones en un sistema más profesionalizado de gestión es una de las opciones que podrían ayudar a superar esta insatisfactoria situación.

Desde nuestro punto de vista, la opción por la fusión debe plantearse acompañada de un proyecto de coedición, de acuerdo con los siguientes objetivos:

- Búsqueda de la eficiencia a través de la colaboración interinstitucional, incluso a nivel internacional.

- Aplicación de un modelo profesionalizado de gestión, dentro de un espíritu de racionalización de recursos humanos y viabilidad económica.

- Fortalecer el compromiso institucional hacia las publicaciones por encima del "amateurismo".

La coedición entre diferentes instituciones es una fórmula de colaboración presente en muchas publicaciones. Es difícil dar datos sobre su extensión, ya que esta característica puede que no se refleje siempre en los directorios y catálogos. Pueden producirse cambios no reflejados en el registro del ISSN o en la ficha catalográfica que responde a lo declarado en el primer número de la publicación.

En ocasiones la coedición puede significar un reparto de roles: una institución puede realizar la labor de distribución editorial (publisher) mientras otra gestiona los contenidos (editor). Por ejemplo, la Revista de historia económica fue editada inicialmente por el Instituto Figuerola de Historia Económica de la Universidad Carlos III de Madrid. A partir de 2006 este centro mantiene la responsabilidad editorial pero la gestión y distribución es asumida por Cambridge University Press, ampliando su título con la fórmula Journal of Iberian and Latin American economic history (RHE-Jilaeh). Sin embargo, mantiene el antiguo ISSN para la edición impresa y utiliza un nuevo código para la electrónica adscribiéndola al editor británico.

\section{http://turan.uc3m.es/uc3m/inst/LF/RHE/index.htm/}

Algunos casos de coedición proceden de fusiones, habituales entre revistas científicas. Una fusión puede ser de especial interés para las revistas que no hayan podido situarse en los primeros cuartiles de los rankings de factor de impacto, como estrategia para poder mejorar su posición.

Para que se produzca un proyecto de fusión es preciso que, al menos, haya dos colectivos que sientan la necesidad de romper las inercias y tengan publicaciones de características y temática similares. Como afirma Armando Laffón (2004) respecto a los proyectos de fusión de las revistas española y mexicana de reumatología": "Los autores que tienen un buen original tienden, como es lógico, a mandarlo a una revista que les puntúe y que tenga una lectura más universal (...). Siguiendo el ejemplo de las revistas de otras sociedades científicas, hoy incluidas en Index medicus, se planteó la fusión de revistas como única estrategia viable y prometedora (...). Sin intentos de cambio, nuestra revista está condenada al anonimato para siempre". La fusión es, sobre todo, una ocasión para redimensionar y profesionalizar las estructuras de trabajo: "Precisará un corrector de estilo y un documentalista. El comité editorial deberá tener un elevado peso específico y una relevancia internacional. Los revisores deberán estar suficientemente preparados en sus respectivas áreas de conocimiento. La nueva publicación deberá ser más citada (hay estrategias para ello), y su distribución tendrá que ser lo más amplia posible. La puntualidad en la salida de cada número será obligatoria" (Laffón, 2004).

\section{Se trata de hacer una revista más com- petitiva, evitar la multiplicación de es- fuerzos, no malgastar fondos y recursos y fomentar la profesionalización}

Ejemplos similares de colaboración iberoamericana se han producido en otras especialidades médicas como parasitología (Alcuino, 2009). También en el caso europeo, PérezPadilla (2006) cita dos ejemplos de revistas de neumología resultado de la fusión entre títulos anteriores. En los Journal Citation Reports $(J C R)^{8}$ se recogen los cambios producidos en los títulos. En la edición de ciencias, el único ejemplo de los últimos años que es resultado de la integración de títulos diferentes ya presentes en WoS, es: Animal. The international journal of animal biosciences, coeditada por British Society of Animal Science (BSAS), Institut National de la Recherche Agronomique (INRA) y la European Federation of Animal Science (EAAP), a partir de la integración en 2007 de tres títulos anteriores: Animal science, Animal research y Reproduction, nutrition, development. Por el contrario, otros ejemplos son tan sólo casos de reducción de series en un único nombre: Quarterly journal of experimental psychology (2006), o Electronics and communications in Japan (2009).

http://journals.cambridge.org/action/displayJournal?jid=ANM

También dentro de los títulos presentes en los JCR, el caso más reciente de fusión de títulos diferentes en ciencias sociales es la publicación Series: desde 2010 es editada por 
Springer (publisher), la Asociación Española de Economía (editor), con el patrocinio de la Fundación SEPI. Su puesta en marcha es resultado de la fusión de dos de las más reconocidas revistas españolas de economía: Spanish economic review e Investigaciones económicas. El cambio realizado ha supuesto la aparición de un nuevo título, pero cuya difusión se apoya en la alta valoración de los anteriores, asegurando su permanencia entre las fuentes de la Web of science y los Journal citation reports.

http://www.asesec.org/sea/series.html

http://www.funep.es/invecon/sp/sAims_Scope.asp

Para potenciar la imagen de continuidad una opción en la fusión es adoptar un título casi idéntico a los previos. Ejemplo de ello es Ciudad y territorio: estudios territoriales, creada en 1992 por la integración de Ciudad y territorio: revista de ciencia urbana (1969-92) y Estudios territoriales (1981-92), y manteniendo la numeración de la publicación más antigua. Otro caso de éxito es el de la revista Estudios geográficos, actualmente editada por el CSIC y, en su momento, resultado de la fusión de la revista que llevaba ese mismo título y que editaba el Instituto Juan Sebastián Elcano desde 1940, y la revista Geographica publicada (entre 1956 y 1986) por el Depto. de Geografía Aplicada de la Univ. de Zaragoza. La fusión de ambas revistas coincidió con un momento de estructuración de departamentos e institutos que trabajaban en el ámbito de la geografía. Estudios geográficos es actualmente una de las revistas españolas mejor posicionadas en su área $y$, en palabras de sus responsables ${ }^{9}$, aunque la experiencia fue en un principio traumática, se ha visto con el tiempo que esa unión fortalecía la revista resultante.

\section{Mediante la fusión se lograrían revistas} más sólidas

Algunos editores conscientes de la necesidad de aunar esfuerzos se empiezan a plantear ya la unión de algunas revistas (normalmente, dos títulos en uno). Sin embargo, este tipo de restructuración parece ser un tema tabú, del que no se habla abiertamente, lo que denota la poca predisposición del sector editorial en su conjunto para abordar ese profundo cambio que parece necesitar.

La fusión de revistas, que puede plantearse como una coedición, con responsabilidad compartida por parte de dos instituciones, presenta algunas ventajas:

- La nueva revista evita el inconveniente de presentarse como "una revista más". Su difusión en bibliotecas y bases de datos es inmediata, no debe empezar una andadura totalmente nueva sino que los cambios que conlleva son los mismos que precisa una revista cuando cambia de título.

- Sólo sumando las contribuciones anuales de cada título, permite aumentar el número de artículos anuales, manteniendo el rigor en la evaluación y, consecuentemente, el número de autores y revisores implicados.

- Si se aumenta la cantidad de números publicados al año, se acortarán los tiempos de proceso, evitando que los autores pasen por largas esperas para ver publicados sus artículos. La rapidez en la publicación de una revista de buena calidad es un reclamo indudable para los autores.

- Garantiza una mayor difusión, aunque sólo fuera sumando los suscriptores de los títulos anteriores.

- Es un medio para superar la imagen de endogamia. Cuantas más instituciones se hallen implicadas, mayor credibilidad se genera sobre su objetivo global y demostrando que no pretende servir de vehículo de transmisión para un grupo determinado.

- Se facilita la incorporación posterior de otros posibles editores que carezcan de revista propia, y que difícilmente pueden incorporarse a una publicación que tuviera una imagen fuertemente institucional.

- Permite dar un salto cualitativo en la investigación publicada: más selección, más filtros. La principal garantía de calidad es el apoyo de un colectivo de evaluadores amplio y plural. La fusión permite reducir el número de personas en la dirección y aumentar el colectivo de revisores.

- Racionalización de gastos y esfuerzos: la fusión puede permitir asumir el gasto de una secretaría profesionalizada y la puesta en marcha de un sistema electrónico de gestión editorial; este requiere de un esfuerzo inicial que difícilmente puede asumir el editor que es único responsable de la revista y, sin embargo, en una situación de estas características se puede abordar con mayor facilidad y representa una oportunidad para agilizar la gestión editorial.

- Amplias posibilidades para la internacionalización: enfoque europeo, enfoque iberoamericano, enfoque global. La unión de dos títulos -y dos equipos editoriales, un sistema de gestión editorial, etc.- permitirán modificar la política editorial, adaptándola a las necesidades de la ciencia española en cuanto a internacionalización, posibilitando la participación en los comités de expertos extranjeros que puedan, además, promocionar la revista en sus países, haciéndola visible en otros lugares gracias a una buena presencia en internet, etc.

- Mejor posicionamiento de partida que una nueva publicación que parte de cero. Si se trata de revistas ya seleccionadas por los índices de citas, esto se tiene en cuenta habitualmente. Si no lo están, aumentan las opciones de ser seleccionadas al poder apoyarse en su historia previa. - Si la revista tiene gestión comercial, la fusión permitiría aumentar la tirada, incrementar el número de anunciantes y consecuentemente generar ingresos. Se podría aprovechar el cambio para replantear el modelo de negocio, algo que por inercia y por la dependencia de los fondos públicos no se suele abordar.

Como posibles inconvenientes pueden considerarse:

- La fusión puede ser un nuevo maquillaje: un reparto formal de tareas sin auténtica responsabilidad común.

- El control real puede permanecer en pocas manos, que se limiten a reproducir esquemas tradicionales sin implicar la colaboración de sus instituciones.

- Para las directivas de una institución o sociedad científica que antes controlaban los contenidos de su publicación, significa una pérdida de autonomía sobre la política editorial y los contenidos de la revista. Esto es positivo, pero en la práctica podría conllevar una sensación de alejamiento, generar desconfianza o falta de interés por la revista 
resultante y animar a que estas directivas inicien nuevas aventuras editoriales.

- La coordinación entre entidades distantes precisa cierto grado de relación personal y de compromiso estable y fuerte entre las instituciones que respaldan la publicación. Cuando las direcciones de los organismos cambian, se corre el riesgo de que estos lazos se rompan o debiliten.

- La fusión de títulos, si no son de características idénticas, puede conllevar una pérdida de especificidad en la cobertura o en sus objetivos. La unión de revistas debería ir precedida de un proyecto de cambio para la revista resultante, precisamente para aprovechar las ventajas de las dos pre-existentes, al mismo tiempo que se innova con el objetivo de convertirla en una publicación mejor.

- Hay riesgo de que las alianzas se produzcan principalmente entre revistas y editoriales comerciales multinacionales como vía para la pervivencia. Si la empresa asume el peso en las decisiones políticas posteriores, puede devenir en cambios sustanciales de su contenido y en un debilitamiento del sector editorial español. Esto está ocurriendo ya incluso con revistas que no son resultado de una fusión (Aréchaga, 2011).

\section{Reflexiones finales}

La fusión de publicaciones es una alternativa adecuada para superar el modelo de revista imperante en HHyCS, hacerla más competitiva, evitar la multiplicación de esfuerzos, no malgastar fondos y recursos, fomentar la profesionalización en las revistas existentes y para crear, en definitiva, revistas más sólidas. Es, por tanto, una oportunidad para plantear la necesaria profesionalización de las funciones más técnicas, lo que puede traducirse en empleo para los graduados en documentación. Es una estrategia de especial interés para las publicaciones que aspiren a la excelencia.

Con esta propuesta no se persigue sumarse a tendencias neoliberales como las fusiones de bancos o empresas que se justifican por una mejora del beneficio económico. El sector editorial en España y en estas disciplinas no actúa movido por el ánimo de lucro, pero no por ello debe ser ajeno a una gestión eficaz de los recursos humanos y materiales de los que se dispone; más aún teniendo en cuenta que un alto porcentaje de la edición científica de revistas está financiada con fondos públicos. Se trataría, más bien, de crear publicaciones serias y sólidas, alejadas de personalismos y del interés por contar con cifras institucionales destacadas, y competitivas desde el punto de vista científico. Esta mejora del sector debería llevar consigo una inversión económica: no una inversión añadida, sino un mejor aprovechamiento de los fondos procedentes de la reordenación del sector. El funcionamiento voluntarista de muchas revistas -cuyos editores tienen la mejor de las voluntades y han puesto su mejor esfuerzo- supone sacar adelante las revistas con recursos institucionales, simplemente porque éstas no tienen presupuestos que les permitan hacer frente a todos los gastos que genera una publicación. La profesionalización de la gestión implica la dotación de recursos humanos y económicos suficientes que garanticen la estabilidad, la calidad y el rigor en la edición de la revista. La financiación para ello puede venir del ahorro por el abandono definitivo de los costes de la edición impresa y, sobre todo, por la racionalización del gasto.

La profesionalización de la gestión implica la dotación de recursos humanos y económicos suficientes que garanticen la estabilidad, la calidad y el rigor en la edición de la revista

La actual dinámica de sobreproducción de revistas de HHyCS es un círculo vicioso del que resulta difícil escapar. No sólo se trata de mejorar la calidad de los contenidos, es también una cuestión de imagen y de una gestión más eficaz de los recursos. Hace falta un cambio estructural, un cambio en la cultura científica que precisa apoyarse en publicaciones de alto reconocimiento no sólo a nivel internacional sino también en el ámbito hispanohablante.

\section{Notas}

1. En estos cálculos sobre revistas y facultades de la UCM se ha considerado la Psicología dentro de las Ciencias sociales.

2. Consulta realizada en junio de 2012.

3. http://bddoc.csic.es:8080 en su versión gratuita, http:// bddoc.csic.es:8085 en la de suscripción.

4. Datos de búsqueda realizada a 29/06/2012. Se seleccionó 2010 por considerar que los años siguientes podrían estar aún incompletos por los habituales retrasos en la edición de algunas publicaciones.

5. Este dato hace referencia a publicaciones en las que no se ha podido realizar el cálculo de apertura externa de los autores por diferentes razones: no presentan la afiliación institucional, aún no tienen la antigüedad necesaria para hacer una evaluación completa o bien no se han recibido los ejemplares solicitados para ello.

6. Como es sabido, cuando en el contexto de los índices de impacto se habla de "autocitas" se refiere a citas a la propia revista, no a autocitas de los autores.

7. La fusión de la Revista española de reumatología (RER) con su homóloga Revista mexicana de reumatología se hizo efectiva en 2005 con el nuevo título de Reumatología clínica, actualmente editada por Elsevier. Su actividad actual le ha permitido superar la falta de artículos, publicando 11 números en 2011, y ha cumplido su objetivo de estar recogida en Medline.

http://www.reumatologiaclinica.org

http://www.ncbi.nlm.nih.gov/nlmcatalog?cmd=historysear ch\&querykey $=1$

\section{Acceso a través de Web of knowledge. http://www.accesowok.fecyt.es}

9. Opiniones expresadas en el desarrollo de una reunión de la Asociación de Geógrafos Españoles en 2012.

\section{Bibliografía}

Alcaín-Partearroyo, María-Dolores; Román-Román, Ade- 
laida; Giménez-Toledo, Elea (2008). “Categorización de las revistas españolas de Ciencias sociales y Humanas en RESH". Revista española de documentación científica, v. 31, n. 1, pp. 85-95.

http://redc.revistas.csic.es/index.php/redc/article/view/414/426 http://dx.doi.org/10.3989/redc.2008.v31.i1.414

Alcuino, Héctor (2009). "Revista ibero-latinoamerica de parasitología: nueva etapa de dos revistas de parasitología". Revista ibero-latinoamericana de parasitología, v. 68, n. 1. http://www.socepa.es/revista/spip.php?article4

Anep/Fecyt (2007). Criterios de calidad en la investigación en humanidades. Madrid: Anep/Fecyt.

http://www.ucm.es/BUCM/der/doc18556.pdf

Archambault, Éric; Vignola-Gagné, Étienne; Côté, Grégoire; Lariviére, Vincent; Gingrasb, Yves (2006). "Benchmarking scientific output in the social sciences and humanities: the limits of existing databases". Scientometrics, v. 68, n. 3, pp. 329-342.

http://dx.doi.org/10.1007/s11192-006-0115-z

Aréchaga, Juan (2011). "Los españoles y las revistas científicas... ique editen ellos!". El país, 15 sept.

http://sociedad.elpais.com/sociedad/2011/09/15/ actualidad/1316037621_850215.html

Bordons, María (2004). "Hacia el reconocimiento internacional de las publicaciones científicas españolas". Revista española de cardiología, v. 57, n. 9, pp. 799-802.

http://hdl.handle.net/10261/11564

Borrego, Ángel; Urbano, Cristóbal (2006). “La evaluación de revistas científicas en ciencias sociales y humanidades". Información, cultura y sociedad, n. 14, pp. 11-27. http://www.scielo.org.ar/pdf/ics/n14/n14a02.pdf

Cantó-Alcaraz, Ramón (2008). “Acerca de la calidad científica de la revista y el control de la endogamia". Ricyde (Revista internacional de ciencias del deporte), v. 4, n. 10.

http://www.cafyd.com/REVISTA/ojs/index.php/ricyde/ article/view/100/86

Casals-Carro, María-Jesús (2011). "Presentación”. Estudios sobre el mensaje periodístico, v. 17, n. 1, pp. 9-14.

http://www.ucm.es/info/emp/Numer_17-1/2-01Presentacion.pdf

Cetto, Ana-María; Alonso-Gamboa, José-Octavio; Córdoba, Saray; Giménez-Toledo, Elea; Chávez-Sánchez, Guillermo. "Organized access to the Ibero-American quality journals: the PPL experience". Scholarly and research communication (en prensa).

Delgado-López-Cózar, Emilio; Jiménez-Contreras, Evaristo; Ruiz-Pérez, Rafael; López-Herrera, Antonio-Gabriel; Gacto-Colorado, María-José; Torres-Salinas, Daniel; Dela-Moneda-Corrochano, Mercedes; Ruiz-Baños, Rosario; Pérez-Ortega, Juan-Manuel; Bailón-Moreno, Rafael; Poyatos-Huertas, Encarnación; Rodríguez-Gálvez, María-José (2005). "InRecs: índice de impacto de las revistas españolas de ciencias sociales". Biblio 3W: revista bibliográfica de geografía y ciencias sociales, v. 10, n. 574.

http://www.ub.edu/geocrit/b3w-574.htm
Editor de Formación universitaria (2012). “Ética de los editores". Formación universitaria, v. 5, n. 3, p. 1.

http://www.scielo.cl/pdf/formuniv/v5n3/art01.pdf http://dx.doi.org/10.4067/S071850062012000300001

Esteban-Navarro, Miguel-Ángel; Novales-Araluce, Ana; Arquero-Avilés, Rosario; Lamarca-Langa, Genaro; MorenoVernis, Miguel; Salvador-Oliván, José-Antonio; GordilloAcosta, Ignacio (2005). "Evaluación de la calidad de las revistas científicas españolas en humanidades y ciencias sociales". Boletín de la Anabad, v. 55, n. 1-2, pp. 377-391.

Guédon, Jean-Claude (2011). "Between quality and excellence; from nation to region: strategies for Latin American scholarly and scientific journals". En: Cetto, Ana-María; Alonso-Gamboa, José-Octavio (comps.). Calidad e impacto de la revista iberoamericana. México: Latindex, Unam, pp. 21-45.

http://www.latindex.unam.mx/librociri

Giménez-Toledo, Elea; Román-Román, Adelaida; AlcainPartearroyo, María-Dolores (2007). “From experimentation to coordination in the evaluation of Spanish scientific journals in the humanities and social sciences". Research evaluation, v. 16, n. 2, pp. 137-148.

http://dx.doi.org/10.3152/095820207X220409

Giménez-Toledo, Elea; Román-Román, Adelaida; Perdiguero, Pablo; Palencia, Irene (2009). "The editorial boards of Spanish scholary journals. What are they like? What should they be like?" The journal of scholarly publishing, v. 40, n. 3, pp. 287-306.

http://dx.doi.org/10.3138/jsp.40.3.287

Laffón, Armando (2004). “Fusión de las revistas española y mexicana de reumatología: un vehículo para conseguir incluirnos en el Index medicus". Revista española de reumatología, v. 31, n. 3, p. 115.

http://www.elsevier.es/sites/default/files/elsevier/ $p d f / 29 / 29 v 31 n 03 a 13060466 p d f 001 . p d f$

Mañana-Rodríguez, Jorge; Giménez-Toledo, Elea (2011). "Coverage of Spanish social sciences and humanities journals by national and international databases". Information research, v. 16, n. 4, paper 506.

http://informationr.net/ir/16-4/paper506.html

Ministerio de Educación (2011). "Resolución de 23 de nov. de 2011, de la Presidencia de la Comisión Nacional Evaluadora de la Actividad Investigadora, por la que se establecen los criterios específicos en cada uno de los campos de evaluación". Boletín oficial del Estado, 30 nov. , pp.127448127461.

http://www.boe.es/boe/dias/2011/11/30/pdfs/ BOE-A-2011-18796.pdf

Morris, Sally (2007). "Mapping the journal publishing landscape: how much do we know?". Learned publishing, v. 20, n. 4, pp. 299-310.

http://dx.doi.org/10.1087/095315107X239654

Olivera-Betrán, Javier (2011). “Tendencias en la edición y en la mejora de la calidad de las revistas científicas españolas de ciencias sociales". Apunts. Educación física y deportes, n. 104 , pp. 5-10. 
http://dx.doi.org/10.5672/apunts.2014-0983.es.(2011/2).104.00

Osca-Lluch, Julia; Mateo-Marquina, María-Elena (2003). "Difusión de las revistas españolas de ciencias sociales y humanidades. Acercamiento bibliométrico". Revista general de información y documentación, v. 13, n. 1, pp. 115-132. http://revistas.ucm.es/index.php/RGID/article/view/ RGID0303120115A/10028

Osca-Lluch, Julia; Haba, Julia; Minguez, Olga; Navarro, Gloria; Velasco, Elena; Salom, Leonardo (2008). “Difusión y factor de impacto nacional e internacional de las revistas científicas españolas". Anales de documentación, v. 11, pp. 145-164.

http://hdl.handle.net/10201/4088

Pérez-Padilla, Rogelio (2006). "La inevitable gestación de revistas médicas multinacionales en América Latina". Revista chilena de enfermedades respiratorias, v. 22, n. 3, pp. 176-180.

http://www.scielo.cl/scielo.php?pid=s071773482006000300005\&script=sci_arttext

Ruiz-Pérez, Rafael; Delgado-López-Cózar, Emilio; JiménezContreras, Evaristo (2006). "Criterios del Institute for
Scientific Information para la selección de revistas científicas. Su aplicación a las revistas españolas: metodología e indicadores". International journal of clinical and health psychology, v. 6, n. 2, pp. 401-424.

http://redalyc.uaemex.mx/src/inicio/ArtPdfRed. jsp?iCve $=33760211$

Torres-Salinas, Daniel; Bordons, María; Giménez-Toledo, Elea; Delgado-López-Cózar, Emilio; Jiménez-Contreras, Evaristo; Sanz-Casado, Elías (2010). "Clasificación integrada de revistas científicas (Circ). Propuesta de categorización de las revistas de ciencias sociales y humanas". El profesional de la información, v. 19, n. 6, pp. 675-682.

http://dx.doi.org/10.3145/epi.2010.nov.15

Torres-Salinas, Daniel; Delgado-López-Cózar, Emilio; Jiménez-Contreras, Evaristo (2009). "Análisis de la producción de la Universidad de Navarra en revistas de ciencias sociales y humanidades empleando rankings de revistas españolas y la Web of science". Revista española de documentación científica, v. 32, n. 1, pp. 22-39.

http://redc.revistas.csic.es/index.php/redc/article/ download/451/463

El Directorio EXIT permite localizar colegas para participar en cursos, conferencias, proyectos, 0 recibir ofertas de trabajo.

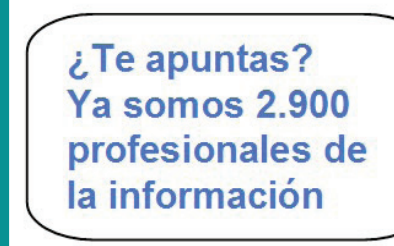

Para bibliotecarios, documentalistas, periodistas, comunicadores, informáticos y diseñadores que ya figuren al menos en Google Scholar, o tengan experiencia ejerciendo en empresas o instituciones. http://directorioexit.info

\section{exit}

Nieves González-Fernández-Villavicencio

Nieves González-Fernández-Villavicencio

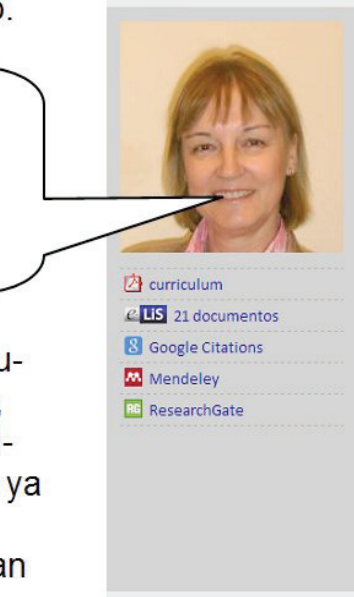

Exit ID: 399

IraLIS: González-Fernández-Villavicencio, Nieves ¿Qué es? Institución: Universidad Pablo de Olavide Área de Biblioteconomía y Documentación Dirección: Ctra. de Utrera, km. 1 P Código postal: 41013 Ciudad: Sevilla Pais: ES - España
Provincia: Sevilla Pais: ES - España Correo-e personal: nievesglez @gnail.con

Correo-e personal: nievesglez @gnail.con
Web institucional: http://www.upo.es $\checkmark$ Dirección válida Web personal: http://www.nievesglez.com $\vee$ Dirección válida Descriptor personal: Social Media Marketing

Especialidades: Biblioteca universitaria; Educación, formación, alfin; Márqueting y promoción Grupos: Recuperación de información y búsquedas; Web 2.0 y redes sociales

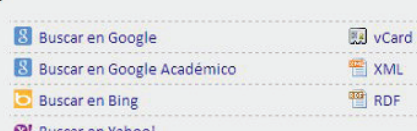

D. Buscar en Bing xML 핌 $\mathrm{RDF}$

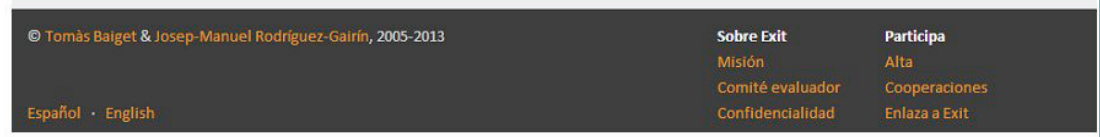

Y acabamos de estrenar nuevo look y nuevas funciones... 